\title{
Numerical analysis of the initial conditions in fractional systems
}

\author{
J. Tenreiro Machado
}

A B S T R A C T

Fractional dynamics is a growing topic in theoretical and experimental scientific research. A classical problem is the initialization required by fractional operators. While the problem is clear from the mathematical point of view, it constitutes a challenge in applied sciences. This paper addresses the problem of initialization and its effect upon dynamical system simulation when adopting numerical approximations. The results are compatible with system dynamics and clarify the formulation of adequate values for the initial conditions in numerical simulations.

Keywords:

Fractional Calculus

Initial conditions

Numerical simulation

\section{Introduction}

Fractional Calculus (FC) was started by the ideas of Leibniz in 1695 and represents the generalization of standard differential calculus up to fractional or complex orders [1-7]. The area remained untouched by applied sciences during several centuries, but in the last years considerable progress took place in the areas of physics, engineering and biology [8-21]. One of the difficulties often found by researchers consists of the initialization of fractional differential equations. In fact, while classical integer order systems require a finite set of initial conditions, fractional operators have an intrinsic memory of the phenomena that is translated into the requirement for a proper initialization and, eventually, to an infinite set of initial conditions [22-38]. The problem becomes even more intricate when we verify that there are several possible definitions for the fractional operators, that may lead to the requirement either of integer or of fractional order initial conditions. Furthermore, a pure mathematical perspective may be too far from the scenario found in the applied world, and analytical approaches may not totally describe the algorithms and restrictions implemented by numerical approximations.

This paper focus the effect of initial conditions upon the response of fractional order dynamical systems (FODS) to be simulated computationally. In this perspective mathematical discussions about integer versus fractional formulations, or physical considerations concerning possible correlations between the values adopted for the initial conditions are not addressed. FODS are simulated by means of algorithms that implement several approximations and, consequently, such issues must be decoupled in order to clarify the effect of each one separately.

Having these ideas in mind, this paper is organized as follows. Section 2 presents the fundamentals of fractional operators and their numerical approximations, formulates the procedure for studying the effect of initial conditions and analyses the results. Finally, Section 3 outlines the main conclusions. 


\section{Fundamental concepts}

There are several definitions of fractional derivatives and integrals of order $\alpha$ [39]. The Riemann-Liouville, Caputo and Grünwald-Letnikov left-sided derivatives are given by:

$$
\begin{aligned}
& \left.{ }_{a}^{R L} \mathcal{D}_{t}^{\alpha} f(t)=\frac{1}{\Gamma(n-\alpha)} \frac{d^{n}}{d t^{n}} \int_{a}^{t} \frac{f(\tau)}{(t-\tau)^{\alpha-n+1}} d \tau, t>a, \operatorname{Re}(\alpha) \in\right] n-1, n[ \\
& { }_{a}^{C} \mathcal{D}_{t}^{\alpha} f(t)=\frac{1}{\Gamma(n-\alpha)} \int_{a}^{t} \frac{f^{(n)}(\tau)}{(t-\tau)^{\alpha-n+1}} d \tau, t>a, n-1<\alpha<n \\
& { }_{a}^{G L} \mathcal{D}_{t}^{\alpha} f(t)=\lim _{h \rightarrow 0}\left[\frac{1}{h^{\alpha}} \sum_{k=0}^{\left[\frac{t-a}{h}\right]}(-1)^{k} \frac{\Gamma(\alpha+1)}{k ! \Gamma(\alpha-k+1)} f(t-k h)\right], t>a, \alpha>0
\end{aligned}
$$

where $\Gamma(\cdot)$ is Euler's gamma function, $[x]$ means the integer part of $x$, and $h$ is the step time increment.

The distinct formulations of fractional derivatives and integrals lead to several proposals for the interpretation of the generalized operator [40-54]. In spite of this diversity it is clear that FODS require an initialization procedure taking into account the history of the dynamical phenomenon that is not asked for in the classical integer order approach. This problematic has been the subject of debate during the last years, but a clear analysis of the impact of the initialization during numerical simulations is still lacking and further study is necessary.

In the numerical algorithm for calculating $\mathcal{D}_{t}^{\alpha}$ often is adopted the Grünwald-Letnikov formulation where the time increment $h$ is approximated by the sampling period $T_{s}$ and the series is truncated at the $r$ th term yielding:

$$
\mathcal{D}^{\alpha}[f(t)] \approx \frac{1}{T_{s}^{\alpha}} \sum_{k=0}^{r}(-1)^{k} \frac{\Gamma(\alpha+1)}{k ! \Gamma(\alpha-k+1)} f\left(t-k T_{s}\right)
$$

Other techniques adopt the Laplace or the $Z$ transforms and lead to rational fractions in the $s$ or $z$ domains. These approximations produce recursive expressions in discrete time, involving both input and output variables, but are not adopted in this study in order to tackle more clearly the initialization variables [55-57,54]. The objective of this paper is to investigate numerically the effect of the initial conditions upon the time responses. For that purpose three test FODS of order $0<\alpha<2$ are considered:

$$
\begin{aligned}
& \mathcal{D}^{\alpha} x+a x=u \\
& \mathcal{D}^{\alpha} x+a x+b x^{3}=u \\
& \mathcal{D}^{\alpha} x+a \sin (x)=u
\end{aligned}
$$

These expressions correspond in the mechanical domain to a fractance and a linear spring $(\mathcal{F L})$, a fractance and a nonlinear spring $(\mathcal{F N})$ and a fractional pendulum $(\mathcal{F P})$.

The FODS are excited by a unit impulse

$$
u(t)= \begin{cases}1 & t=0 \\ 0 & t \neq 0\end{cases}
$$

For analysing the effect of initial conditions is considered a statistical approach, that can be outlined as follows:

1. Definition of the parameters of the FODS and values of $\alpha$

2. Repeat the following two steps for $i=1, \ldots, N$ independent experiments

(a) Generation of the initial conditions $x_{i}(t), t<0$, in series (4), obeying a given probability distribution function (pdf)

(b) Numerical simulation of the FODS

3. Calculation of standard deviation $\sigma_{x}$ versus $t$ of the dynamical responses $x_{i}(t), t \geqslant 0, i=1, \ldots, N$

It should be noted that the standard deviation $\sigma_{x}(t)$ of the dynamic responses measures the variation between the numerical solutions, themselves, due to the different initial conditions. The index does not captures the deviation between the analytical solution and the numerical approximations. Therefore, numerical errors are not included in the comparisons.

In the sequel we adopt $N=500, T_{s}=0.001$ and $r=1000$. For $0<\alpha<1$ a fractional integration of order $\alpha$ based on the Grünwald-Letnikov truncated series is adopted. On the other hand, for $1<\alpha<2$ a standard integration of order 1 followed by the numerical approximation of order $\alpha-1$ is used.

We start by "stimulating" the initial conditions with a Gaussian pdf with zero average and standard deviation $\sigma_{0}$. This procedure does not addresses issues of physical meaning and has in mind solely producing a sample to be described statistically. By other words, considerations about the time correlation of the successive numerical values adopted for the initial 
conditions are not included in the tests. This approach may be, in one hand, somewhat unrealistic and may introduce values considerably noisy, but, on the other hand, guarantees independence from additional considerations of physical or numerical nature.

Figs. 1 and 2 show the locus of standard deviation $\sigma_{x}$ versus $(\alpha, t)$ of the numerical solution of $\mathcal{F L}(5), a=1$, for $\sigma_{0}=0.01$ and $\sigma_{0}=0.1, t<0$, in the initialization pdf, to be denoted cases 1 and 2, respectively. Figs. 3 and 4 , to be denoted cases 3 and 4, repeat the experiment with the $\mathcal{F N}(6),(a, b)=(1,1)$, and the $\mathcal{F P}(7), a=1$, respectively, for $\sigma_{0}=0.1$ and $t<0$ in the initialization pdf. We observe that, as one should expect, the integer orders are not sensitive to the initial conditions contrary to the fractional case, and that the effect of the perturbations tend to dissipate as time evolves. Furthermore, we verify that

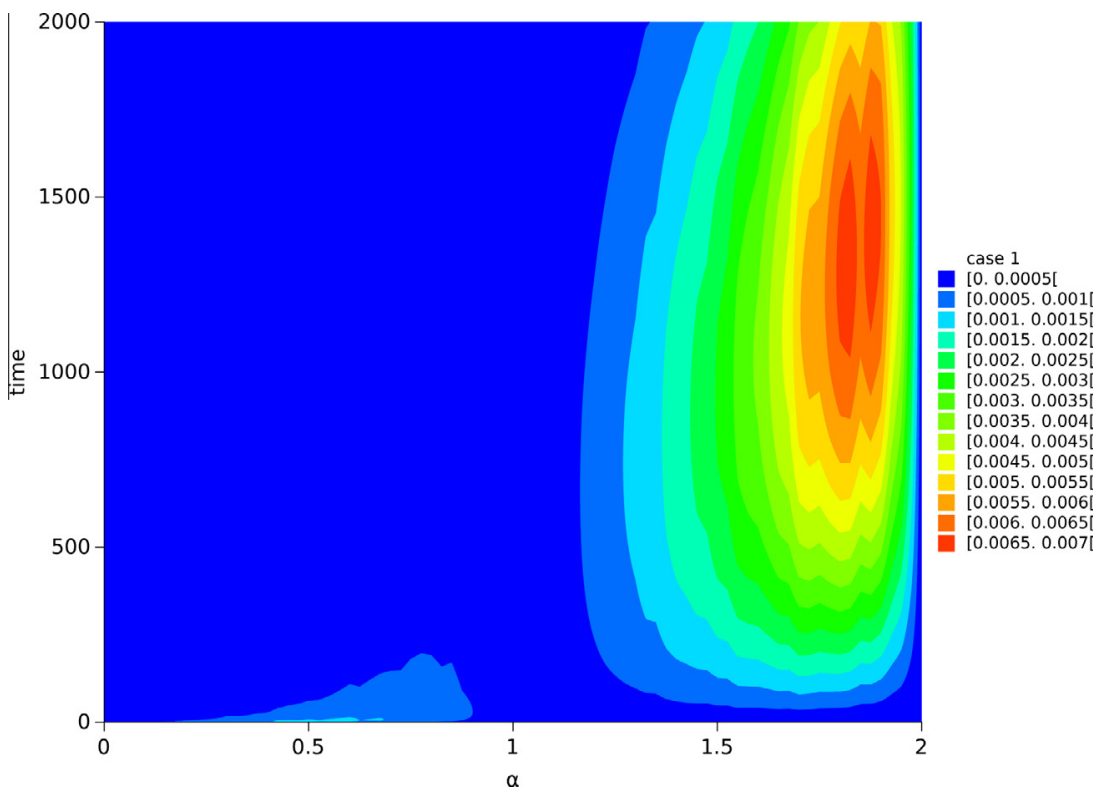

Fig. 1. Locus of $\sigma_{x}$ versus $(\alpha, t)$ for the numerical solution of $\mathcal{F L}(5)$ and $\sigma_{0}=0.01, t<0, r=1000$ (case 1 ).

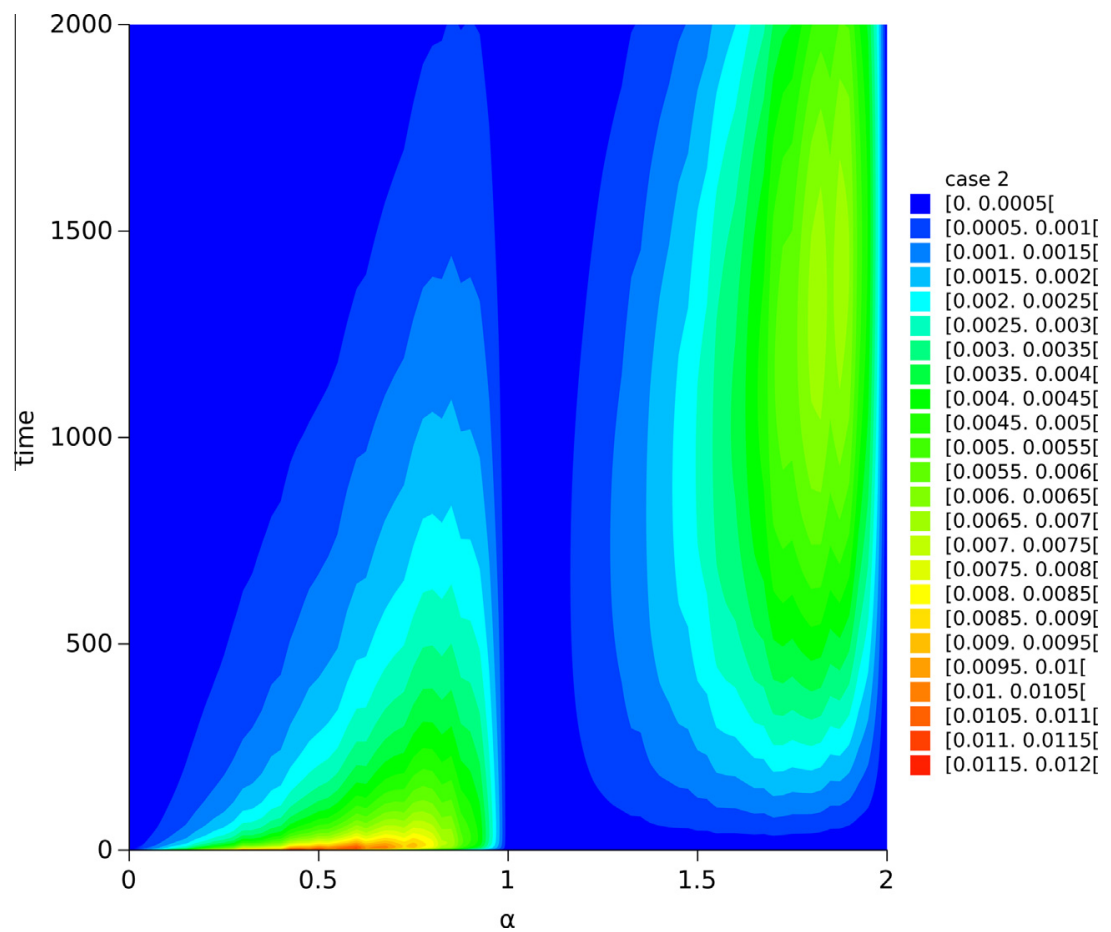

Fig. 2. Locus of $\sigma_{x}$ versus $(\alpha, t)$ for the numerical solution of $\mathcal{F L}(5)$ and $\sigma_{0}=0.1, t<0, r=1000$ (case 2 ). 


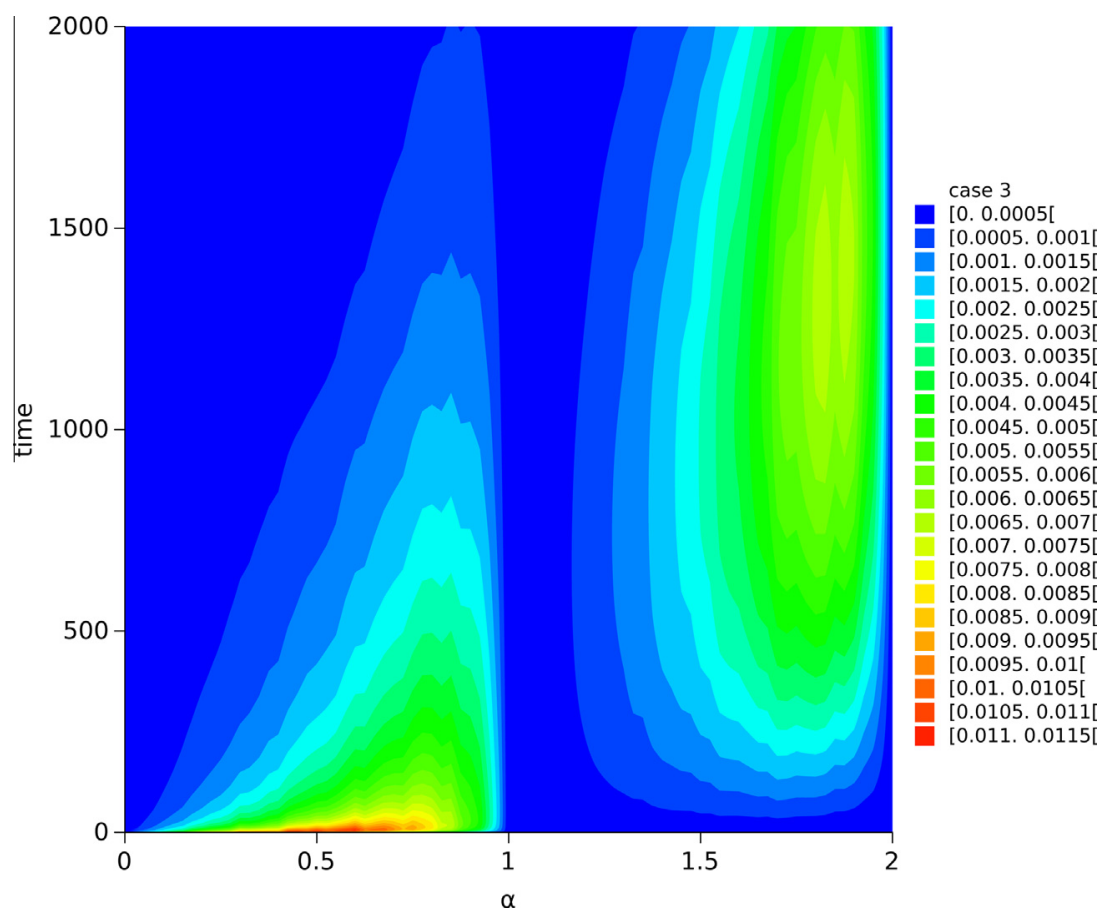

Fig. 3. Locus of $\sigma_{x}$ versus $(\alpha, t)$ for the numerical solution of $\mathcal{F N}(6)$ and $\sigma_{0}=0.1, t<0, r=1000$ (case 3).

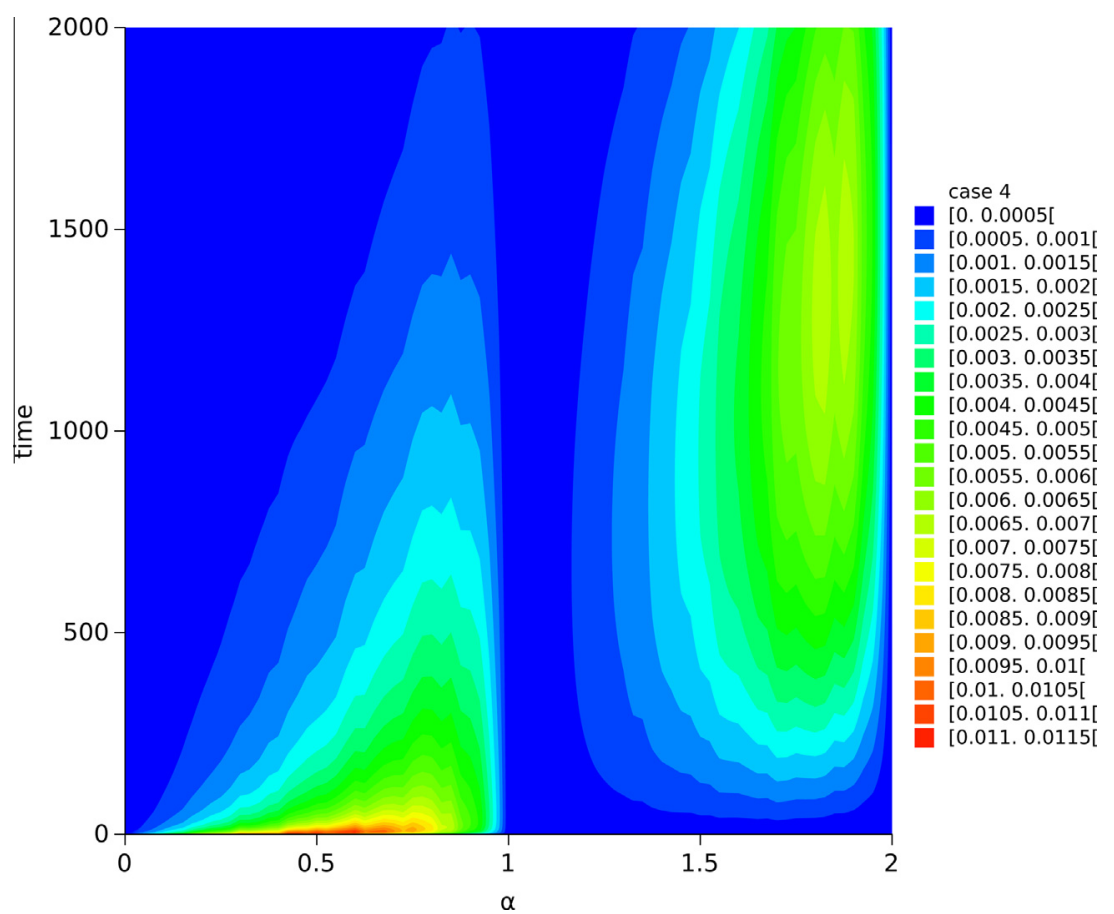

Fig. 4. Locus of $\sigma_{x}$ versus $(\alpha, t)$ for the numerical solution of $\mathcal{F P}(7)$ and $\sigma_{0}=0.1, t<0, r=1000$ (case 4).

the time evolution of $\sigma_{x}$ is distinct for $0<\alpha<1$ and $1<\alpha<2$. This is due to the range of $\alpha$ that leads to decaying response, in the first case, and oscillatory behaviour, in the second. On the other hand, there is no significant difference between the behaviour for linear and the non-linear systems in what concerns the sensitivity to the initial conditions. Experiments with other values of $b$ in (6) lead to similar conclusions. It is interesting to note that the higher amplitudes of $\sigma_{x}$ occur for $t \approx 0$ and $\alpha=0.5$; however, the dissipation is slower for systems with $0.5<\alpha<1$ which leads to a slower decaying in $\sigma_{x}$. 
Two other experiments were now designed for testing the effect of the initial conditions. For feeding the initial conditions in (4) is considered the Gaussian pdf, with zero average, but having a linear variation of the standard deviation $\sigma_{0}(t)$ between the extreme instants $t=-r T_{s}$ and $t=0$ of the series. For the analysis is tested the linear system $\mathcal{F} \mathcal{L}(5)$ and $\sigma_{0}(t)=0.1 \frac{t}{r T_{s}}$, or $\sigma_{0}(t)=0.1\left(\frac{t}{r T_{s}}+1\right), t<0$, for increasing, or decreasing, amplitudes of the standard deviation (to be denoted as cases 5 and 6). Figs. 5 and 6 depict the corresponding results.

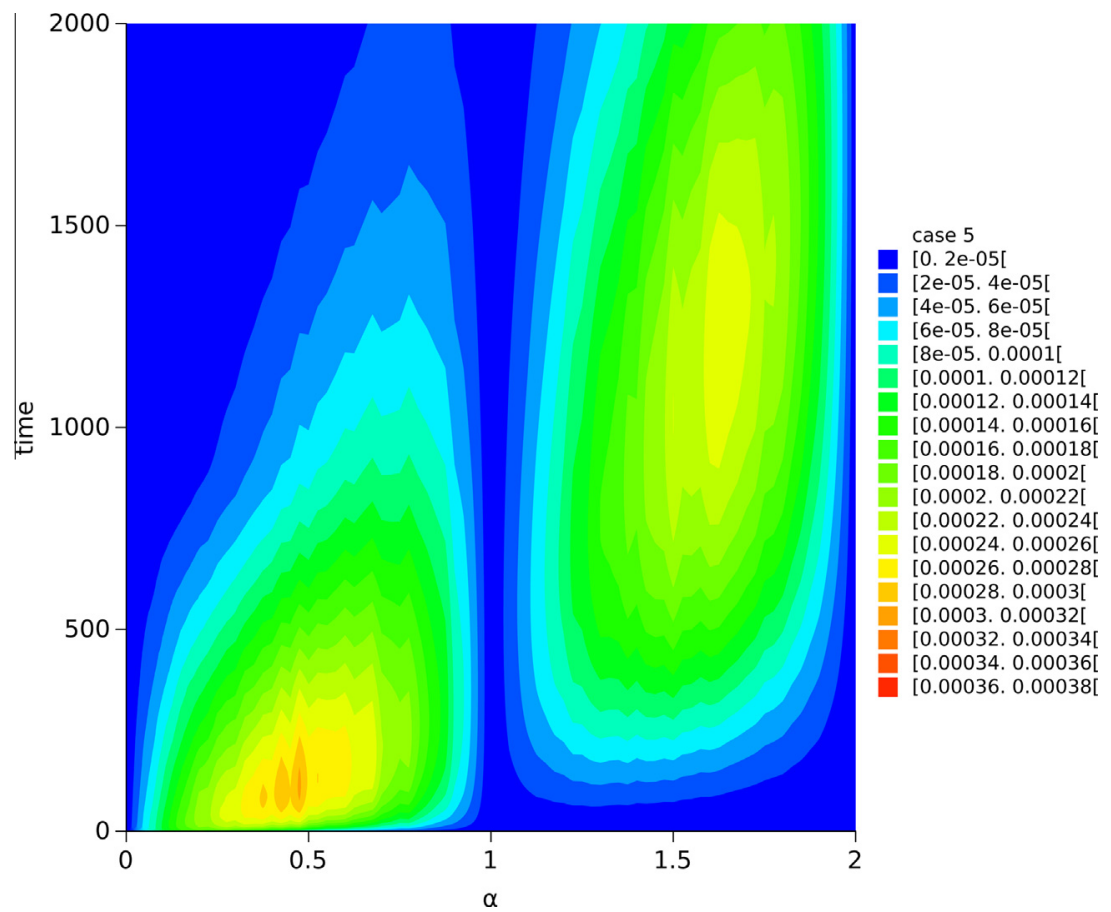

Fig. 5. Locus of $\sigma_{x}$ versus $(\alpha, t)$ for the numerical solution of $\mathcal{F} \mathcal{L}(5)$ and $\sigma_{0}(t)=0.1 \frac{t}{r T_{s}}, t<0, r=1000$ (case 5).

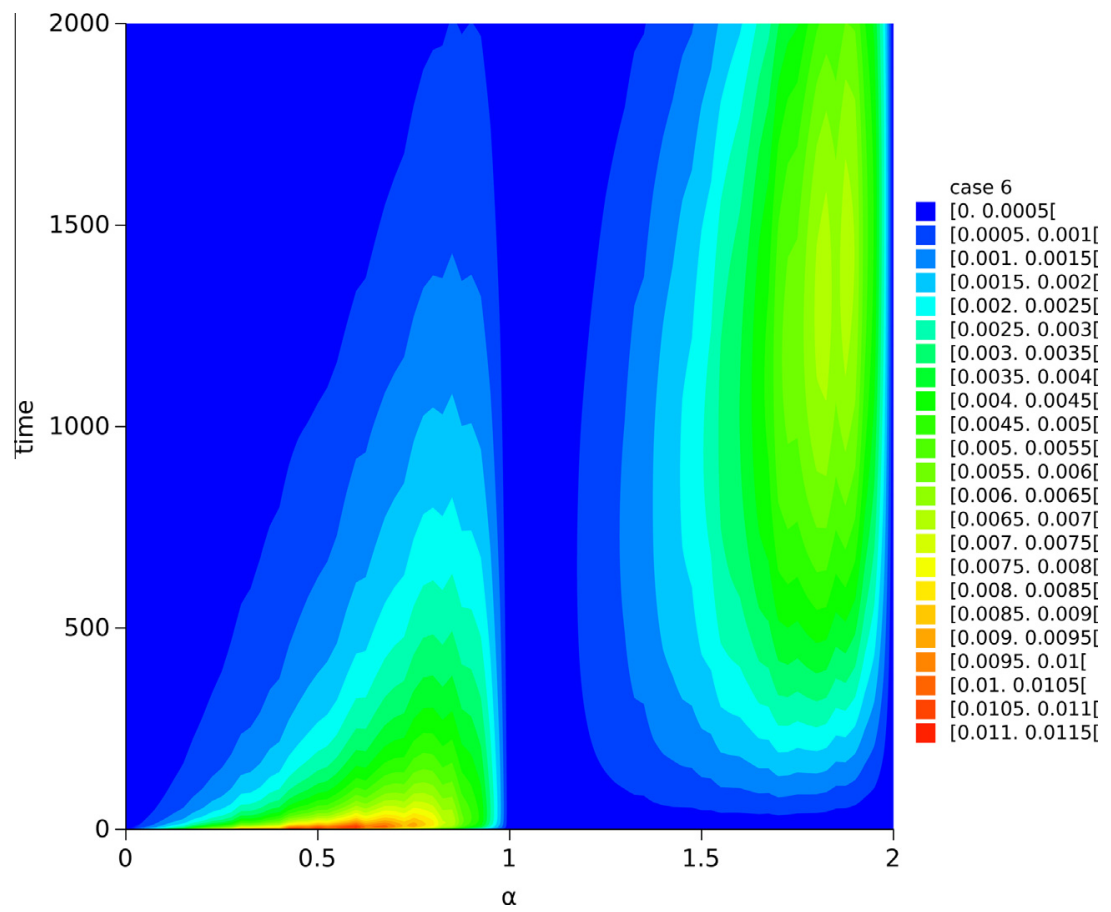

Fig. 6. Locus of $\sigma_{x}$ versus $(\alpha, t)$ for the numerical solution of $\mathcal{F} \mathcal{L}(5)$ and $\sigma_{0}(t)=0.1\left(\frac{t}{r T_{s}}+1\right), t<0, r=1000($ case 6$)$. 


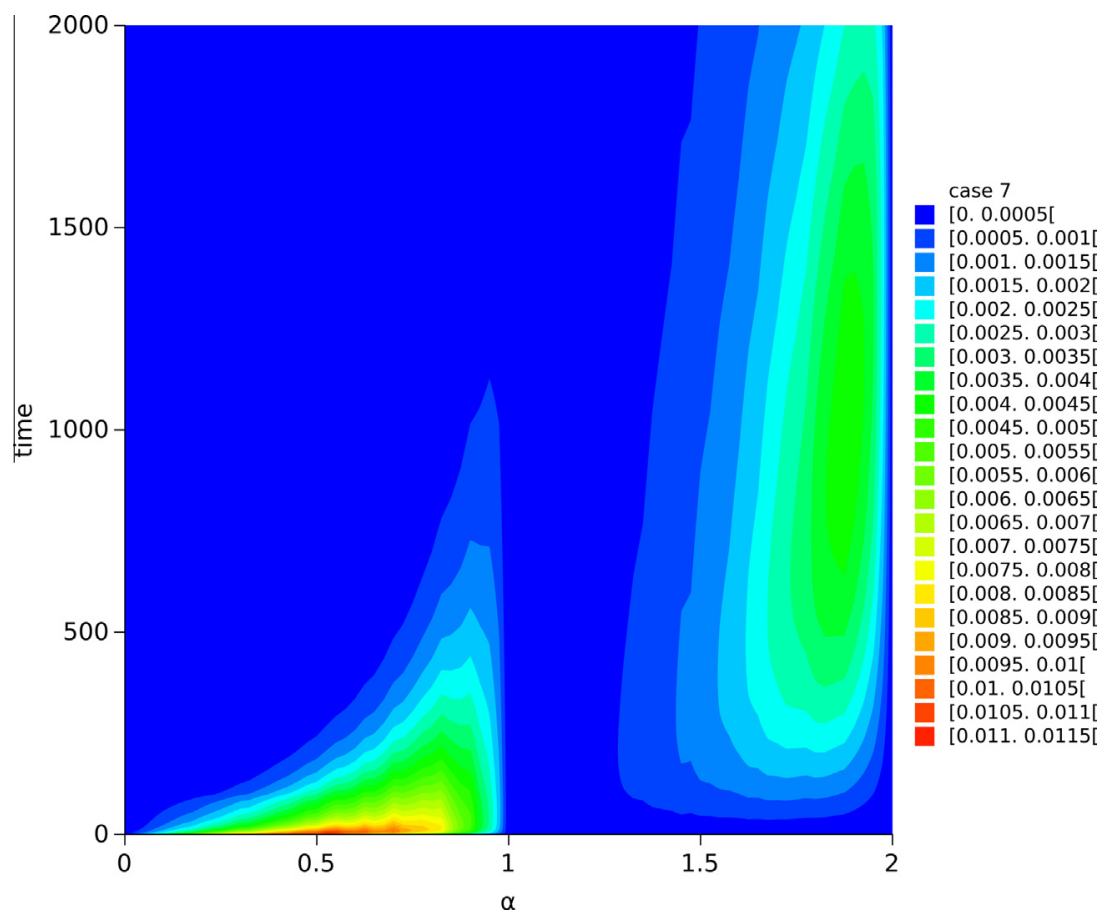

Fig. 7. Locus of $\sigma_{x}$ versus $(\alpha, t)$ for the numerical solution of and the $\mathcal{F} \mathcal{L}(5)$ and $\sigma_{0}=0.1, t<0, r=100$ (case 7).

We observe that case 5 produces deviations $\sigma_{x}$ close to case 1 , with fixed $\sigma_{0}$, while case 6 leads to deviations inferior to all previous experiments. These results demonstrate that the "recent" initial conditions (i.e., near to $t=0$ ) have an higher effect upon the responses $x(t)$ than the "old" ones (i.e., near the region $t=-r T_{s}$ ).

Another aspect to be discussed consists on the effect of the truncation order $r$ in (4) since it is the numerical representation of the memory effect. Therefore, the experiment of case 2 is repeated for a smaller truncation order, namely for the and the $\mathcal{F} \mathcal{L}(5)$ and $r=100$ (to be denoted as case 7), producing the locus depicted in Fig. 7. We verify that a lower value of $r$ means having a smaller memory content and, consequently, a smaller effect over $\sigma_{x}$.

In conclusion, the proposed method of analysis leads to results consistent with intuitive knowledge in dynamical systems. There is no mathematical proof for all possible cases of fractional order systems when adopting experiments restricted to a finite set of numerical tests. Nevertheless, the scheme is straightforward to implement and, therefore, can by applied with different dynamical systems for evaluating the effect of approximations in the initialization.

\section{Conclusions}

In this paper the problem of initial conditions from the viewpoint of numerical approximations was analysed. While the majority of research as focused on the interpretation, or the analytical derivation of initial conditions for fractional differential equations, the present study embraces the computational implementation by means of truncated series. In this line of thought experiments may prove to be too stressful for dynamical system simulation, since no physical restrictions were considered for number generation, but experiments constitute critical benchmarks that encompass practical cases. The numerical tests were performed in a statistical perspective and demonstrated that the concepts usually adopted in fractional dynamics are valid and useful.

\section{References}

[1] Oldham K, Spanier J. The fractional calculus: theory and application of differentiation and integration to arbitrary order. New York: Academic Press; 1974.

[2] Samko S, Kilbas A, Marichev O. Fractional integrals and derivatives: theory and applications. Amsterdam: Gordon and Breach Science Publishers; 1993.

[3] Miller K, Ross B. An introduction to the fractional calculus and fractional differential equations. New York: John Wiley and Sons; 1993.

[4] Podlubny I. Fractional differential equations. An introduction to fractional derivatives, fractional differential equations, to methods of their solution. Mathematics in science and engineering, vol. 198. San Diego: Academic Press; 1998.

[5] Kilbas A, Srivastava H, Trujillo J. Theory and applications of fractional differential equations. North-Holland mathematics studies, vol. 204. Amsterdam: Elsevier; 2006.

[6] Petráš I. Fractional-order nonlinear systems: modeling, analysis and simulation. Heidelberg: Springer; 2011.

[7] Zhou Y. Basic theory of fractional differential equations. World Scientific; 2014.

[8] Oustaloup A. La commande CRONE: commande robuste d'ordre non entier. Paris, France: Hermès; 1991. 
[9] Carpinteri A, Mainardi F, editors. Fractals and fractional calculus in continuum mechanics. Wien and New York: Springer-Verlag; 1997.

[10] Hilfer R. Application of fractional calculus in physics. Singapore: World Scientific; 2000.

[11] Westerlund S. Dead matter has memory! Kalmar, Sweden: Causal Consulting; 2002.

[12] West B, Bologna M, Grigolini P. Physics of fractal operators. New York: Springer; 2003.

[13] Zaslavsky G. Hamiltonian chaos and fractional dynamics. Oxford: Oxford University Press; 2005.

[14] Magin R. Fractional calculus in bioengineering. Redding: Begell House Inc.; 2006.

[15] Sabatier J, Agrawal OP, Machado JT. Advances in fractional calculus: theoretical developments and applications in physics and engineering. Dordrecht, The Netherlands: Springer; 2007.

[16] Tarasov V. Fractional dynamics: applications of fractional calculus to dynamics of particles, fields and media. New York: Springer; 2010.

[17] Diethelm K. The analysis of fractional differential equations: an application-oriented exposition using differential operators of caputo type, series on complexity, nonlinearity and chaos. Heidelberg: Springer; 2010.

[18] Mainardi F. Fractional calculus and waves in linear viscoelasticity: an introduction to mathematical models. London: Imperial College Press; 2010.

[19] Baleanu D, Diethelm K, Scalas E, Trujillo JJ. Fractional calculus: models and numerical methods, series on complexity, nonlinearity and chaos. Singapore: World Scientific Publishing Company; 2012.

[20] Valério D, da Costa JS. An introduction to fractional control. Stevenage: IET; 2012.

[21] Ionescu C. The human respiratory system: an analysis of the interplay between anatomy, structure, breathing and fractal dynamics. Series in bioengineering. London: Springer-Verlag; 2013.

[22] Bagley RL. The initial value problem for fractional order differential equations with constant coefficients. Tech. Rep. AFIT-TR-EN-88-1 (2nd ed.), Air Force Institute of Technology, Glenn Research center; (September 1989).

[23] Lorenzo CF, Hartley TT. Initialized fractional calculus. Tech. Rep. TP-2000-209943, NASA, Glenn Research center; (February 2000).

[24] Diethelm K, Ford NJ. Analysis of fractional differential equations. J Math Anal Appl 2002;265(2):229-48.

[25] Ortigueira MD. On the initial conditions in continuous-time fractional linear systems. Signal Process 2003;83(11):2301-9.

[26] Diethelm K, Ford N, Freed A, Luchko Y. Computer methods in applied mechanics and engineering. Comput Math Appl 2005;194(6-8):743-73.

[27] Heymans N, Podlubny I. Physical interpretation of initial conditions for fractional differential equations with Riemann-Liouville fractional derivatives. Rheologica Acta 2006;45(5):765-71.

[28] Lorenzo CF, Hartley TT. Initialization of fractional-order operators and fractional differential equations. J Comput Nonlinear Dyn 2008;3(2):021101$1-1-9$.

[29] Sabatier J, Merveillaut M, Malti R, Oustaloup A. How to impose physically coherent initial conditions to a fractional system? Commun Nonlinear Sci Numer Simul 2010;15(5):1318-26.

[30] Deng J, Ma L. Existence and uniqueness of solutions of initial value problems for nonlinear fractional differential equations. Appl Math Lett $2010 ; 23(6): 676-80$.

[31] Luchko YF, Rivero M, Trujillo JJ, Velasco MP. Fractional models, non-locality, and complex systems. Comput Math Appl 2010;59(3):1048-56.

[32] Ortigueira MD, Coito FJ. System initial conditions vs derivative initial conditions. Comput Math Appl 2010;59(5):1782-9.

[33] Rachid M, Maamar B, Said D. Multivariable fractional system approximation with initial conditions using integral state space representation. Comput Math Appl 2010;59(5):1842-51.

[34] Trigeassou J, Maamri N. Initial conditions and initialization of linear fractional differential equations. Signal Process 2011;91(3):427-36.

[35] Herzallah MAE, Baleanu D. Fractional-order variational calculus with generalized boundary conditions. Adv Differ Equ 2011; (Article ID 357580).

[36] Baleanu D, Mustafa OG. On the existence interval for the initial value problem of a fractional differential equation. Hacettepe J Math Stat $2011 ; 40(4): 581-7$.

[37] Hartley TT, Lorenzo CF, Trigeassou J-C, Maamri N. Equivalence of history-function based and infinite-dimensional-state initializations for fractionalorder operators. ASME J Comput Nonlinear Dyn 2013;8(4):041014-1-4-7.

[38] Baleanu D, Bhrawy AH, Taha TM. Two efficient generalized Laguerre spectral algorithms for fractional initial value problems. Abstr Appl Anal 2013; (Article ID 546502)

[39] Valério D, Trujillo JJ, Rivero M, Machado JT, Baleanu D. Fractional calculus: a survey of useful formulas. Eur Phys J Spec Top 2013;222(8):1827-46.

[40] Nigmatullin RR. A fractional integral and its physical interpretation. Theor Math Phys 1992;90(3):242-51.

[41] Rutman RS. On the paper by R.R. Nigmatullin a fractional integral and its physical interpretation. Theor Math Phys 1994;100(3):1154-6.

[42] Tatom FB. The relationship between fractional calculus and fractals. Fractals 1995;3(1):217-29.

[43] Rutman RS. On physical interpretations of fractional integration and differentiation. Theor Math Phys 1995;105(3):393-404.

[44] Adda FB. Geometric interpretation of the fractional derivative. J Fract Calc 1997;11:21-52.

[45] Yu Z-G, Ren F-Y, Zhou J. Fractional integral associated to generalized cookie-cutter set and its physical interpretation. J Phys A: Math Gen 1997:30(15):5569-77.

[46] Moshrefi-Torbati M, Hammond JK. Physical and geometrical interpretation of fractional operators. J Franklin Inst 1998;335(6):1077-86.

[47] Adda FB. Interprétation géometrique de la différentiabilité et du gradient d'ordre réel. CR Acad Sci - Ser I - Math 1998;326(8):931-4.

[48] Kiryakova V. A long standing conjecture failes? In: Rusev P, Dimovski I, Kiryakova V, editors. Transform methods and special functions, varna’96, institute of mathematics and informatics. Windsor, Ontario, Canada: Bulgarian Academy of Sciences; 1998. p. 579-88.

[49] Gorenflo R. Afterthoughts on interpretation of fractional derivatives and integrals. In: Rusev P, Dimovski I, Kiryakova V, editors. Transform methods and special functions, varna'96, institute of mathematics and informatics. Windsor, Ontario, Canada: Bulgarian Academy of Sciences; 1998. p. 589-91.

[50] Mainardi F. Considerations on fractional calculus: interpretations and applications. In: Rusev P, Dimovski I, Kiryakova V, editors. Transform methods and special functions, varna'96, institute of mathematics and informatics. Windsor, Ontario, Canada: Bulgarian Academy of Sciences; 1998 . p. 594-7.

[51] Podlubny I. Geometric and physical interpretation of fractional integration and fractional differentiation. Fract Calc Appl Anal 2002;5(4):367-86.

[52] Machado JAT. A probabilistic interpretation of the fractional-order differentiation. Fract Calc Appl Anal 2003;6(1):73-80.

[53] Stanislavsky AA. Probability interpretation of the integral of fractional order. Theor Math Phys 2004;138(3):418-31.

[54] Machado JAT. Fractional derivatives: probability interpretation and frequency response of rational approximations. Commun Nonlinear Sci Numer Simul 2009;14(9-10):3492-7.

[55] Machado JT. Analysis and design of fractional-order digital control systems. Syst Anal Model Simul 1997;27(2-3):107-22.

[56] Podlubny I. Fractional-order systems and $\mathrm{PI}^{\lambda} \mathrm{D}^{\mu}$-controllers. IEEE Trans Autom Control 1999;44(1):208-13.

[57] Chen Y, Moore K. Discretization schemes for fractional-order differentiators and integrators. IEEE Trans Circuits Syst-I: Fundam Theory Appl 2002;49(3):363-7. 